\title{
Patients experienced a lack of control over their time in hospital
}

Holloway IM, Smith P, Warren J. Time in hospital.J Clin Nurs 1998 Sep;7:460-6.

\section{Question}

What are patients' experiences and perceptions of "time" during a hospital stay?

\section{Design}

Grounded theory.

\section{Setting}

South of England, UK.

\section{Patients}

11 patients of varying ages with different medical conditions who were in hospital in an acute care medical ward.

\section{Methods}

Data were gathered by observing interactions between patients and nurses in hospital. Each patient also participated in an indepth, unstructured interview in his or her home. During the interview, patients were asked to talk freely about their feelings of being in hospital and were prompted for follow up of their ideas. Participants were not directed to talk specifically about their perceptions of "time" while in hospital. Data were systematically coded, categorised, and compared within and across interviews using the technique of constant comparison.

\section{Main findings}

Time was an important problem in the life of patients in hospital. During their hospital stay, patients felt their personal time was lost, normal lives disrupted, ordinary events suspended, and control over time lost. For example, one patient said "Oh, (the days were) unbearably long. The first day it didn't bother me at all, but the second day, that seemed forever...' Patients felt the need to keep track of time, to find ways to pass time, and sought ways to create structure in their days. Patients who were seriously ill felt they could not recall time, lost time, or felt isolated. Patients felt that time passed slowly in hospital, especially during certain periods of the day or night. This often led to feelings of loneliness and depression. Patients found ways of passing and managing their time in hospital, which lessened boredom, alleviated anxiety, and counteracted depression and loneliness. Interactions with other patients, visitors, and health professionals helped to pass time, were appreciated, and increased patients' quality of life. Patients tried to assist in their healthcare management and to look well, with the goal of decreasing the length of their hospital stay. Patients were aware that although they perceived themselves as having too much time, health professionals experienced a lack of time.

\section{Conclusions}

Patients felt that their lives were disrupted while in hospital and that precious personal time was lost. During their hospital stay, patients sought control over time, tried to keep track of time, and found ways of passing time. Patients realised that time was a scarce resource for health professionals; this was a direct contrast to the sense of "too much time" experienced by themselves.

Source of funding: no external funding.

For correspondence: Dr I M Holloway, Institute of Health and Community Studies, Bournemouth University, Royal London House, Christchurch Road, Bournemouth BH1 3LT,UK. Fax +44 (0) 1202 504194.

\section{Commentary}

This study by Holloway et al reinforces the value of qualitative research that aims to describe the experience of being ill. The authors have provided thought provoking insight into the world of patients in hospital by focusing on the importance of time. This study provides evidence that time is an important facet of the hospital experience and confirms the findings of other authors who have investigated similar themes. ${ }^{12}$ Holloway et al provide nurses with various issues for consideration in practice and future research.

Indepth interviews with a small sample of people will inevitably provide a rich range of opinions and experiences. It was interesting in this study to see that certain experiences seem to be shared among patients in hospital, such as feeling bored and isolated. Although these insights are not new, the study helps to put them in context and should encourage nurses to question their relevance to their own client groups. Also, this study encourages consideration of how to minimise time that is wasted by long waiting periods in clinics.

It would be helpful to have known more about the nature of the different illnesses involved. For instance, time might be more precious to patients with advanced cancer who want to use their remaining time as productively as possible.

Nurses often seek to improve their practice by relating interventions to the needs of patients. They can help people to cope with the demands that illness places on their sense of time through the use of relatively simple strategies. For example, the authors suggest that nurses can empower patients to have control over their day by abolishing some of the rituals, by tailoring care to individual needs (eg, allowing patients to choose at what time they get up), by providing clocks and calendars to reduce disorientation, by introducing patients to one another to help to pass the time, and by involving them in planning their discharge to reduce uncertainty about the length of stay. The findings should also encourage nurses to investigate this important issue further among other patient populations.

\section{Daniel Kelly, RN, RNT, MSc \\ Senior Nurse, Research and Development University College London Hospitals NHS Trust London, UK}

1 Tishelman C, Sachs L. The diagnostic process and the boundaries of normality. Qual Health Res 1998;8:48-60.

2 Knafl KA, Deatrick JA. How families manage chronic conditions: an analysis of the concept of normalization. Res Nurs Health 1986;9:215- 